\title{
On an Attempt to Formulate an Optimization Model for Maximizing the Radio Media Advertising Reach in Indian Scenario
}

\author{
P. C. Jha \\ Department of Operational \\ Research \\ University of Delhi, \\ Delhi, India
}

\author{
S. P. Singh \\ Department of Management \\ Studies \\ IIT Delhi \\ Delhi, India
}

\author{
Remica Aggarwal \\ MIT-SOER, MIT-ADT University, \\ Pune, India
}

\begin{abstract}
Present research work is an application based research. It focuses on maximizing the total reach which can be accumulated by using different forms of radio media. The problem is further optimized under the constraints of budget and maximum -- minimum number of advertisements etc. Chance constrained goal programming approach has been used to derive the optimal reach under the event of uncertainty.
\end{abstract}

\section{Keywords}

Radio advertising; ISM methodology; Digital age; Media-mix advertising Optimization Problem

\section{INTRODUCTION}

As far as share of radio media sector is concerned, radio industry is a very small part of the broadcasting universe. Radio, as a medium gains from best of both worlds i.e. the multiplicity of genres as well as diversity of languages ${ }^{1-5}$.

This paper focuses on optimizing the advertising reach captured by using radio media in today's digital age. In this research paper, a mathematical approach has been presented to study the radio media selection and optimization problem associated with maximizing their reach. The aim is to find the number of advertisements that could be placed for a product in different radio media options so as to maximize the uncertain advertising reach and effectiveness amongst the potential adopters of the product. Multiple segments considered are the various zones of India such as the north zone, south zone, east and west zone. Factors considered are the advertising rates of radio media in different time intervals. The paper is arranged as follows : Section 2 deals with the model formulation. Section 3 deal with the managerial implications.

\section{LITERATURE REVIEW}

\section{Notations}

$s \quad: 1,2, \ldots . . \mathrm{S}$ segments or Indian radio zones

$m: 1,2, \ldots \mathrm{M}$ media option of radio media like All India radio and $\mathrm{FM}$ radio

$s_{m} \quad$ : insertion/slot in $\mathrm{m}^{\text {th }}$ medium like day slot or evening slot

$r_{s m s_{m}}$ : target consumer reach for an advertisement in $s^{\text {th }}$ segment , $m^{\text {th }}$ media option, $s_{m}^{\text {th }}$ slot/insertion.

$R_{s m s_{m}} \quad$ : number of readers or viewers in $m^{\text {th }}$ media option, $i_{m}{ }^{\text {th }}$ slot/insertion in $s^{\text {th }}$ segment

$C_{s m s_{m}} \quad$ : advertisement cost of inserting one advertisement for the product in $m^{\text {th }}$ media option, $\mathrm{s}_{m}^{\text {th }}$ slot/insertion in $s^{\text {th }}$ segment

$\operatorname{Min}_{s m s_{m}}$ : minimum number of advertisements in different positions for the product in $m^{\text {th }}$ media option, ${s_{m}}^{\text {th }}$ slot/insertion in $s^{\text {th }}$ segment

$\operatorname{Max}_{s m s_{m}}$ : maximum number of advertisements in different positions for the product in $m^{\text {th }}$ media option in $s^{\text {th }}$ segment

$x_{s m s_{m}} \quad:$ decision variable corresponding to $m^{\text {th }}$ media option, $\mathrm{s}_{m}{ }^{\text {th }}$ slot/insertion in $s^{\text {th }}$ segment

$W_{m}$ medium : weight corresponding to age criteria in $m^{\text {th }}$ radio

$P_{s m s_{m}} \quad$ : percentage of people in $s^{\text {th }}$ segment for the product for $m^{\text {th }}$ media option, $s_{m}^{\text {th }}$ slot/insertion in $s^{\text {th }}$ segment

A : Advertising budget

Assumptions

1. A single product for advertising through radio media is considered.

2. Segmentation of consumers is based upon their location i.e. north, south, east and west zone.

3. Further criteria is that of age i.e. radio programmes for adults, children and retired or old age people.

4. There is only one media i.e. radio media having at least two media options. There are media options such as All India radio which is a government run radio channel and also some private radio channels such as FM .

5. There is a fixed advertising or financial budget .

\subsection{Deterministic model}

The problem for finding the optimal number of advertisements to be allocated to different media sources $m$, $m=1,2, \ldots . M$ for the newly introduced FMCG product that would maximize the total advertising reach for all media options of radio media can be formulated as:

$$
\begin{gathered}
\operatorname{Max} Z_{m}=\sum_{d \in D} \sum_{s \in S} \sum_{o_{m} \in O} \sum_{s_{m} \epsilon S} r_{s m s_{m}} x_{s m s_{m}} \quad \forall m \in M \\
\operatorname{Max} Z_{m}=\sum_{s \in S} \sum_{s_{m} \epsilon S}\left(W s_{c m} * P_{s m s_{m}}\right) * R_{s m s_{m}}^{d} x_{s m s_{m}}^{d} \\
\forall s \in S, \forall m \in M, \forall s_{m} \in O
\end{gathered}
$$


Subject to

$$
\begin{aligned}
& \sum_{s \epsilon S} \sum_{s_{m} \epsilon I} C_{s m s_{m}} x_{s m s_{m}} \leq A \\
& x_{s m s_{m}} \geq \operatorname{Min}_{s m s_{m}} \forall s \in S, \quad \forall m \in M, \forall s_{m} \in O \\
& x_{s m s_{m}} \leq \operatorname{Max}_{s m s_{m}} \forall s \epsilon S, \forall m \epsilon M, \forall s_{m} \in O \\
& x_{s m s_{m}} \geq 0 \quad \forall s \epsilon S, \forall m \epsilon M, \forall s_{m} \in O \\
& x_{s m s_{m}} \text { are integers }
\end{aligned}
$$

\subsection{Stochastic model with deterministic equivalents}

Applying chance constraint approach given by [5-7] the deterministic model given in equation (1.1) to equation (1.6) is converted to its stochastic version described from equation (1.7) to equation (1.12) as follows:

$$
=\sum_{d \in D} \sum_{S \in S} \sum_{o_{m} \in O} \sum_{i_{m} \in S} r_{s m s_{m}} x_{s m s_{m}} \quad \forall m \in M
$$

Subject to

$$
\begin{aligned}
& \sum_{s \epsilon S} \sum_{s_{m} \epsilon I} C_{s m s_{m}} x_{s m s_{m}} \leq A \\
& x_{s m s_{m}} \geq \operatorname{Min}_{s m s_{m}}^{d} \forall s \epsilon S, \quad \forall m \epsilon M, \forall s_{m} \in O \\
& x_{s m s_{m}} \leq \operatorname{Max}_{s m s_{m}} \forall s \in S, \forall m \epsilon M, \forall s_{m} \in O \\
& x_{s m s_{m}} \geq 0 \quad \forall s \epsilon S, \quad \forall m \epsilon M, \quad \forall s_{m} \in O
\end{aligned}
$$

Where

$\sum_{s \epsilon S} \sum_{s_{m} \epsilon S}\left(w_{s m s_{m}} * P_{s m s_{m}}\right) * R_{s m o_{m} s_{m}}^{d} x_{s m s_{m}}^{d} \geq$ $F_{A^{m}}^{-1}\left(\alpha^{m}\right) \quad \forall m \in M$

\section{- Deriving advertising reach uncertainty}

To determine the deterministic equivalent of the reach objective the following procedure is adopted. The actual advertising reach may differ from the planned advertising reach due to customer behaviour, market competition, choice of radio media etc. Hence, advertising reach is probabilistic in real practice. The mean and standard deviation data is based on historical evidences. Let $A^{1}$ be the aspired advertising reach for government radio media objective function and $A^{2}$ be the aspired advertising reach for the private radio media objective function. This aspiration level may be obtained by getting the ideal solution of solving both the radio objectives separately. Applying chance constraint approach the probabilistic equations for advertising reach objective functions are written as :

$$
P\left(\sum_{s \in S} \sum_{s_{m} \in S} r_{s m s_{m}} x_{s m s_{m}} \geq A^{m}\right) \geq \alpha^{m}, \quad \forall m \in M
$$

$$
F_{A^{m}}\left(\sum_{s \epsilon S} \sum_{s_{m} \epsilon S} r_{s m s_{m}} x_{s m s_{m}}\right) \geq \alpha^{m}
$$

Which implies

$\sum_{s \epsilon S} \sum_{s_{m} \epsilon S} r_{s m s_{m}} x_{s m s_{m}} \geq F_{A^{m}}^{-1}\left(\alpha^{m}\right) \quad \forall m \epsilon M$

Deterministic equivalent in inequality equation (1.15) preserves the linearity of inequality equation (1.14).

\section{GOAL PROGRAMMIG APPROACH}

The problem formulated in section 4.4 can be rewritten as

$\operatorname{Max} Z_{m}=\sum_{s \epsilon S} \sum_{s_{m} \epsilon S} r_{s m s_{m}} x_{s m s_{m}} \quad \forall m \in M$

Subject to

$\sum_{s \in S} \sum_{s_{m} \epsilon S} C_{s m s_{m}} x_{s m s_{m}} \leq A$

$\sum_{s \in S} \sum_{s_{m} \in S} r_{s m s_{m}} x_{s m s_{m}} \geq F_{A^{m}}^{-1}\left(\alpha^{m}\right)$

$x_{s m s_{m}} \geq \operatorname{Min}_{s m s_{m}} \forall s \in S, \forall m \in M, \forall s_{m} \in S$

$x_{s m o_{m} i_{m}}^{d} \leq \operatorname{Max}_{s m o_{m} s_{m}}^{d} \forall s \in S, \forall m \in M, \forall o_{m} \in O, \forall i_{m} \in I$

$x_{s m o_{m} i_{m}}^{d} \geq 0 \quad \forall s \in S, \forall m \in M, \forall o_{m} \in O, \forall i_{m} \in I$

$x_{s m o_{m} i_{m}}^{d}$ are integers

This problem when solved using standard mathematical programming approach provides infeasible solution. In order to obtain compromise solution, goal programming approach is used to solve the problem defined from equation (1.16) to equation (1.22) in two stages .

\section{Stage 1}

Minimize

$g_{0}(\gamma, \delta, X)=\delta^{d}+\sum_{d \epsilon D} \sum_{s \epsilon S} \sum_{o_{m} \in O} \sum_{i_{m} \epsilon I} \gamma_{S m o_{m} i_{m}}^{d}+$

$\sum_{d \epsilon D} \sum_{s \epsilon S} \sum_{o_{m} \in O} \sum_{i_{m} \epsilon I} \delta_{s m o_{m}}^{\prime d} i_{m}$

Subject to

$\sum_{d \epsilon D} \sum_{s \epsilon S} \sum_{o_{m} \in O} \sum_{i_{m} \epsilon I} C_{s m o_{m} s_{m}} x_{s m o_{m} i_{m}}+\gamma-\delta=A$

$x_{s m s_{m}}+\gamma_{s m s_{m}}-\delta_{s m s_{m}}=0 \quad \forall s \epsilon S, \forall m \epsilon M, \forall s_{m} \epsilon O$

$x_{s m s_{m}}+\gamma_{s m s_{m}}^{\prime}-\delta_{s m s_{m}}^{\prime}=0 \quad \forall s \in S, \forall m \in M, \forall s_{m} \epsilon O$ (1.26)

$x_{s m s_{m}} \geq 0 \quad \forall s \in S, \quad \forall m \in M, \forall s_{m} \in O$

$x_{s m s_{m}}$ are integers

Let $\left(\gamma^{0}, \delta^{0}, X^{0}\right)$ be the optimal solution for the problem equation (1.23) to equation (1.28). Where $\gamma_{s m o_{m} i_{m}}$ and $\delta_{s m o_{m} i_{m}}$ are the over and under-achievement (negative and positive deviational) variables of the goals for their respective objective/constraint function for lower bound constraints and $\gamma_{s m o_{m} i_{m}}^{\prime}$ and $\delta^{\prime}{ }_{s m o_{m}} i_{m}$ are the over and under-achievement (negative and positive deviational) variables of the goals for their respective objective /constraint function of upper bound constraints.

Stage 2

Minimize

$g(\gamma, \delta, X)=\sum_{m=1}^{M} \varphi_{m} \gamma_{m}$

Subject to

$$
\begin{aligned}
& \sum_{s \epsilon S} \sum_{s_{m} \epsilon S} r_{s m s_{m}} x_{s m s_{m}}+\gamma_{m}-\delta_{m}=F_{A^{m}}^{-1}\left(\alpha^{m}\right) \\
& \forall m \in M \\
& g(\gamma, \delta, X)=g_{0}\left(\gamma^{0}, \delta^{0}, X^{0}\right)
\end{aligned}
$$

Along with constraints from (1.24)-(1.28)

Here $\varphi_{m}$ denotes $\mathrm{m}^{\text {th }}$ objective function weights. $\gamma_{m}$ and $\delta_{m}$ denotes the positive and negative deviational variables respectively corresponding to the $m^{\text {th }}$ objective function ( for $m=1,2 \ldots M)$. 


\section{LITERARY RECCOMMENDATIONS}

Success through radio media can be achieved through the following literary suggestions and recommendations .

- Knowing your target audience : It is important to know your target audience to be appropriate . Making a list of radio station helps. Radio stations also offers programs you want to know more about before you buy.

- lot of radio listening (LRL) : It is required to pay attention to what catches your ear, and what passes you by.

- $\quad$ Serious casting (SC) : So, you have a great script. Voice actors are consummate professionals and want to give you just what you pay for.

- Good Production Is Essential [GP] :Unlike television commercials, production is more simple for a radio commercial. Your copy isn't relying on any visuals, so it's vital you capture the listener's attention from the start.

- Find the most affordable rates [AR] : Take advantage of the low ad rates for radio. Use your negotiating skills to get a good deal on an advertisement bundle. The more ads you buy, the better rates you'll be able to get.

- Right timing [RT] : Advertisement rates are generally less expensive in the first and third quarters. more, the seasonality of the ad buy can influence your creative approach, making the selling message even more powerful.

- Radio advertising cost [ RAC]: The -night spots because the radio audience tends to peak during commuting hours.

- Choice of radio station [CRS] : Your target market should determine the type of station you choose to advertise on. Buying Radio Time [BRT] : Radio stations design their programming to attract certain listeners, and then sell those listeners to advertisers

- Choose your time slots [ TS] : Not all minutes have the same value, so choose your time carefully. The day is divided not into hours but into day-parts: A.M. drive time, 6 a.m. to 10 a.m., has the most

- Radio prices based on slots : Radio prices are based on the day-part, the length of the spot you run, and the frequency, or

- Writing Radio Copy : You can write your own ad copy or you can hire an agency or have the radio station do it.

- Radio ads require repetition to work : you won't be reaching any one group often enough to motivate them to take action.

Note :* Psychologically speaking, the average person needs to hear an advertising message three times per week for it to be memorable. That's called a "Three Frequency." Knowing what we know about radio listening habits and average time spent listening, a message must be played about 21 times per week to reach the average listener three times.

* Reach and frequency are opposing forces in the radio advertising realm. They compete with each other for dominance.

Success $=21$ Ads per Week, 52 Weeks per Year per Station. In radio lingo, we call that a $21 / 52$ schedule, or branding.

\section{REFERENCES}

[1] Saini , R. 2013. A historic perspective of community radio in India , International journal of informative and futuristic research , 1(4), pp97-100.

[2] Pavarala , V. 2011. Community Radio in India: Redefining the Media Landscape, Society \& Culture, Center for the advanced study of India, University of Pennsylvania.

[3] Media Sector Analysis Report 2019, https://www.equitymaster.com/research-it/sectorinfo/media/Media-Sector-Analysis-Report.asp

[4] Study on listenership, reach and effectiveness of community radio stations in India , Ministry of Information and broadcasting, Government of India , 2018.

[5] Charnes, A. , Cooper, W. , DeVoe, J.K., Learner, D.B and Reinecke, W. 1968. A goal programming model for media planning. Management Science. 14: 422-430.

[6] Charnes, A. and Cooper , W. 1959. Chance-constrained programming. Management Science, 5: 73-79.

[7] Charnes, A. and Cooper , W. 1963. Deterministic equivalents for optimizing and satisfying under chance constraints", Operations Research , 11: 18-39. 